\title{
A MODEL OF THERMAL ENERGY STORAGE ACCORDING TO THE CONVENTION OF BOND GRAPHS (BG) AND STATE EQUATIONS (SE)
}

\author{
Marian Cichy, Prof. \\ Jacek Kropiwnicki, Ph. D. \\ Zbigniew Kneba, Ph. D. \\ Gdańsk Univeristy of Technology, Poland
}

\begin{abstract}
The main advantage of the use of the Bond Graphs method and State Equations for modeling energy systems with a complex structure (marine power plants, hybrid vehicles, etc.) is the ability to model the system components of different physical nature using identical theoretical basis. The paper presents a method of modeling thermal energy storage, which is in line with basic BG theory. Critical comments have been put forward concerning multiport energy storage introduced by other authors or the so-called C-field. In suggested approach, the decision not to use pseudo Bond Graphs has been justified as not being in line with basic BG theory. On the basis of molecular physics it was considered that the state variable, in physical and mathematical sense, should be temperature rather than entropy. Examples of the application of the proposed approach to thermodynamic processes and heat exchange have been presented. The application of a single graph as a model for thermal energy storage has been illustrated by a way of numerical simulation examples.
\end{abstract}

Keywords: Energy systems modeling; Bond Graphs method

\section{INTRODUCTION}

In complex energy systems heat exchange elements are used to determinate the total efficiency and the impact of the systems on the environment $[7,19]$. Usage of mathematical modeling and numerical simulation for construction improvement and energy saving, creates the need for a unified and comprehensive modeling of the entire system $[2,3,8$, $14,16,17,18,20,21,22]$. The method, which enables the realization of such tasks is the method of BG and SE.

In basic BG theory applied in problems in the area of mechanics, hydraulics, electricity, power stations, marine power plants and complex energy systems $[4,5,11,26,28,30]$ the following graphs with single nodes are used as "external" model elements:

- Sources of effort and sources of flow,

- Kinetic and potential energy storage,

- Elements that dissipate energy (dissipating elements).

Elements with multiport nodes are one nodes, zero nodes and energy converters in the form of transformers and gyrators.

The application of this basic theory to thermal processes has encountered difficulties from the start. These difficulties are mainly related to the failure to find an answer to following questions: Which parameter does the accumulation of energy represented or in other words, which parameter can be considered to be the state variable in mathematical sense? Does the accumulated thermal energy constitute potential energy or kinetic energy? Does the introduction of the concept of "resistance" [11,27,28] to the modeling of thermal processes represent the dissipation of energy or its degradation? The lack of answer to these questions led to the introduction of so-called pseudo bond graphs (PBG) $[4,10,11,12,25,26,27$, 29] in parallel with real bond graphs. This was a considerable departure from the basic theory, in which the unit of the product of generalized effort and generalized flow is the unit of power. Further elements, which have been specially introduced to the thermal process models are double nodes of energy storage and of thermal resistance as well as the graph defined by a double pair of energetic parameters $[4,21,26]$.

The graph representing the thermal energy storage adopted by the cited authors is shown in figure 1 .

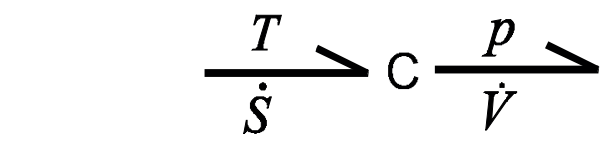

Fig. 1. BG model of thermal energy storage adopted in papers [11, 28, 30]

The energy storage model in the form as shown in figure 1 does not fit the general BG theory. General BG theory concerns energy storage element as an 1-port type element, which fulfills the following relation: 


$$
\sum_{i=1}^{N} e_{i} f_{i}=0
$$

On the basis of molecular physics $[1,6,13,24]$, it is possible to state that the only form of accumulating thermal energy in gases is the kinetic energy of molecules, which is exclusively dependent on temperature. In solid bodies where intermolecular forces play an important role, molecules form oscillators that store kinetic and potential energy [26]. The heat capacity in constant-volume of solid bodies is dependent on temperature and is determined on the basis of Debye theory $[6,13,24]$.

The basic objective of this paper is to show that the models of thermal processes in the form of BG can be created by solely applying elements incorporated in basic BG theory. This means, that the introduction of 2-port type energy storage element and 2-port type thermal resistance element into the modeling of thermal processes, pseudo bond graphs in particular, is not expedient and just complicates the modeling of complex energy systems that require a uniform approach to modeling the elements of the system, in which the processes of a physical nature occur. An example of such a system is a model of an internal combustion engine described in the paper [8].

\section{THE PROPOSAL OF A NEW APPROACH TO THE MODELING OF THERMAL PROCESSES}

As already stated in the proposed approach, solely the elements of the BG are applied that have been incorporated in the modeling of mechanical, hydraulic and electrical processes. Temperature rather than entropy [23] has been adopted as the state variable defining the storage of thermal energy. In basic BG theory, the graph representing thermal energy storage is a 1-port type element $[8,9]$. The speed of accumulation is defined by the relation:

$$
\frac{\mathrm{d} U}{\mathrm{~d} t}=\dot{E}_{A}=\frac{\mathrm{d}\left(C_{v} m T\right)}{\mathrm{d} t}
$$

It was assumed that $\mathrm{R}$ element is 1-port type element and represents energy loss process, due to irreversible energy transfer to the atmosphere. The problem of dissipation element $\mathrm{R}$ will be illustrated by example in this chapter below. State equations are determined on the basis of the relation obtained directly from the graphical model:

$$
\dot{E}_{A}=\sum_{i=1}^{N} \dot{E}_{i}
$$

On the basis of molecular physics $[1,6,13,24]$, it is possible to state that the only form of accumulating thermal energy in gases is the kinetic energy of molecules, which is exclusively dependent on temperature. In solid bodies where intermolecular forces play an important role, molecules form oscillators that store kinetic and potential energy [26]. The heat capacity in constant-volume of solid bodies is dependent on temperature and is determined on the basis of Debye theory $[6,13,24]$.

The basic objective of this paper is to show that the models of thermal processes in the form of BG can be created by solely applying elements incorporated in basic BG theory. This means, that the introduction of 2-port type energy storage element and 2-port type thermal resistance element into the modeling of thermal processes, pseudo bond graphs in particular, is not expedient and just complicates the modeling of complex energy systems that require a uniform approach to modeling the elements of the system, in which the processes of a physical nature occur. An example of such a system is a model of an internal combustion engine described in the paper [8].

\section{THE PROPOSAL OF A NEW APPROACH TO THE MODELING OF THERMAL PROCESSES}

As already stated in the proposed approach, solely the elements of the BG are applied that have been incorporated in the modeling of mechanical, hydraulic and electrical processes. Temperature rather than entropy [23] has been adopted as the state variable defining the storage of thermal energy. In basic BG theory, the graph representing thermal energy storage is a 1-port type element $[8,9]$. The speed of accumulation is defined by the relation:

$$
\dot{\mathbf{T}}=f_{T}(\mathbf{T}, \mathbf{U})
$$

where:

$\mathrm{T}$ - the vector of the temperatures of individual elements of the system,

$\mathrm{U}$ - the vector the independent functions of time characterizing the energy fluxes in the system under consideration (control vector).

The energy sources in the thermal processes adopted in the paper are shown on figure 2. It should be noted, that in the " $\mathrm{b}$ " type source, both energetic parameters may be independent functions of time. A new development is adopting source "c", in which the thermal flux is an independent function of time. Such source is present in a feedwater heater or a microwave oven, for example. Energy converters (fields) have been introduced (fig. 2) without the need to declare their type; whether they are a transformer or gyrator. Solely the basic condition (1) is fulfilled, which means:

$$
e_{\text {in }} f_{\text {in }}=e_{\text {out }} f_{\text {out }}
$$

Relations between parameters pointed in equation (5) can be either linear or nonlinear. 
Energy Sources

$$
\begin{aligned}
& \mathrm{SE}_{\mathrm{T}} \frac{T}{\dot{S}} \quad T=T(t) \quad \text { a) }
\end{aligned}
$$

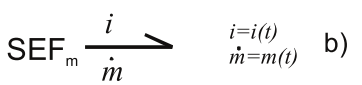

$$
\begin{aligned}
& \mathrm{SF}_{\mathrm{Q}} \frac{e_{E}}{f_{E}} \quad e_{e_{\bar{E}}} Q(\dot{t}) \\
& \mathrm{SF}_{\mathrm{v}} \frac{p}{\dot{V}} \longrightarrow \quad \dot{V}=V(t) \quad \mathrm{d}
\end{aligned}
$$

Energy converters

$\stackrel{T_{1}}{\dot{S}_{1}} \mathrm{PE}_{\mathrm{sS}} \frac{T_{2}}{\dot{S}_{2}}$ a)

$\stackrel{i_{1}}{\dot{m}_{1}} \longrightarrow \mathrm{PE}_{\mathrm{MS}} \frac{T_{2}}{\dot{S}_{2}}$ b)

$\stackrel{T_{1}}{\dot{S}_{1}} \mathrm{PE}_{\mathrm{SM}} \frac{i_{2}}{\dot{m}_{2}} \mathrm{c}$ )

$\left.\stackrel{p_{1}}{\dot{V}_{1}} \mathrm{PE}_{\mathrm{vs}} \frac{T_{2}}{\dot{S}_{2}} \mathrm{~d}\right)$

Fig. 2. Energy sources and converters used in the proposed modeling method

The problem of energy transducers and dissipation element $\mathrm{R}$ will be illustrated by example of en electrical circuit with resistor shown on fig. 3 .

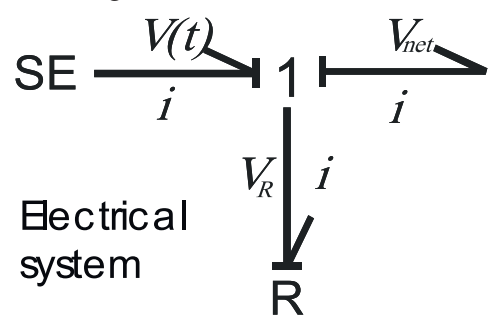

Fig. 3. Electrical circuit with resistor

R element transforms electrical energy to thermal energy. A real resistor is a thermal energy storage element with it's properties: mass, specific heat, area which exchanges heat with the ambient. A resistor model should respect those parameters and have the possibility to calculate temperature by some different ways of heat exchange with the ambient. According to our proposal, using energy transducer and heat dispersing element allows to construct a model which joins electrical and thermal elements (fig. 4).

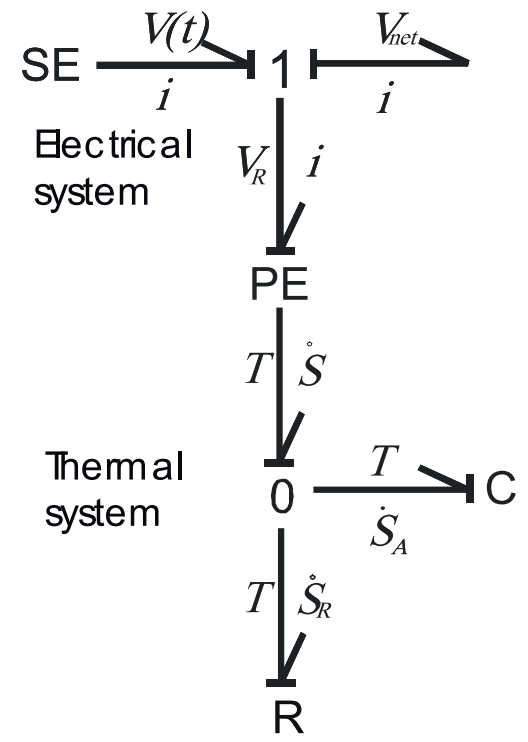

Fig. 4. Model which joins electrical and thermal elements
PE element transforms electrical energy to thermal energy, however $\mathrm{R}$ element disperses this energy to the ambient. There is no doubt that our model allows better processes simulation which are taking place in above example. It is obvious, that dissipating energy flux during steady state $\left(\dot{\mathrm{S}}_{\mathrm{A}}=0\right)$ is equal $\mathrm{TS}_{\mathrm{R}}=\mathrm{V}_{\mathrm{R}} \mathrm{i}$.

In the following paragraphs application examples of this approach will be presented as well as numerical simulations using BG elements shown on figure 2 and state equations.

\section{BG MODEL OF THERMAL ENERGY STORAGE IN THERMODYNAMIC PROCESSES}

An illustration of the application of the proposed approach to modeling of thermal energy storage in a thermodynamic process is the process of the change in volume $\dot{V}(t)$ shown diagrammatically on figure 5 of a defined mass of gas $m$, to which a thermal flux is fed $\mathrm{Q}(\mathrm{t})$. The $\mathrm{BG}$ model of this process using the elements from figure 2 is shown on figure 6 .

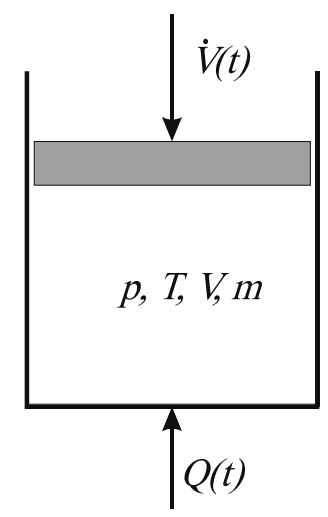

Fig. 5. Diagram of the thermodynamic process under consideration

The control vector in the state equation (4) for the BG model on figure 4 is:

$$
\mathbf{U}=\left[\begin{array}{l}
\dot{V} \\
Q
\end{array}\right]
$$

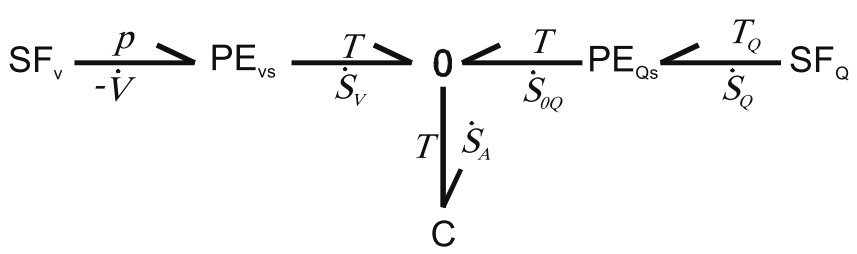

Fig. 6. Model of the thermodynamic process in the form of a bond graph

The basic state equation in accordance with the general relation (3) directly obtained from the BG model on figure 6 is:

$$
T \dot{S}_{A}=-p \dot{V}+T_{Q} \dot{S}_{Q}
$$


In accordance with the adopted definitions:

$$
T \dot{S}_{A}=\frac{d U}{d t} \text { and } T_{Q} \dot{S}_{Q}=Q
$$

the equation (7) represents the first law of thermodynamics. After incorporating relation (2) in the relation (7) and applying the Clapeyron equation, the relation (7) will be transformed into the general form of a state equation (4), which in the case under consideration is the form of:

$$
\dot{T}=-\frac{R}{C_{V}} * \frac{\dot{V}(t)}{V(t)} T+\frac{Q(t)}{C_{V} m}
$$

\section{NUMERICAL EXAMPLE OF A SIMULATION OF THE THERMODYNAMIC PROCESS}

The example concerns the compression process with a thermal flux being fed. Oxygen has been adopted as the medium $\left(\mathrm{R}=259.8 \mathrm{~kJ} / \mathrm{kgK}, \mathrm{C}_{\mathrm{v}}=0.66 \mathrm{~kJ} / \mathrm{kgK}\right)$. The adoption of a constant value for specific heat does not change the equation (8). The initial conditions for $\mathrm{t}=0$ :

$$
\mathrm{p}_{\mathrm{p}}=105 \mathrm{~Pa} ; \mathrm{T}_{\mathrm{p}}=290 \mathrm{~K} ; \mathrm{V}_{\mathrm{p}}=10^{-2} \mathrm{~m}^{3} .
$$

From the state equation, the mass of the medium $\mathrm{m}=1.33$ $10^{-2} \mathrm{~kg}$. The course of the change in volume has been taken to be in the form of a function:

$$
V=A_{V} \cos \omega t+A_{V}+V_{k} \text { for } 0 \leq t \leq \tau / 2
$$

In the calculations, it has been accepted that: $\mathrm{V}_{\mathrm{k}}=10^{-3} \mathrm{~m}^{3} ; \tau=0.1 \mathrm{~s}$. Based on this data the following was calculated:

$\omega=\frac{2 \pi}{\tau}=31.4 \mathrm{rad} / \mathrm{s} ; \quad A_{V}=\frac{V_{p}-V_{k}}{2}=4.5 \cdot 10^{-3} \mathrm{~m}^{3}$

The speed of the change in volume for $t \in\langle 0, \tau\rangle$ is expressed by way of the relation:

$$
\dot{V}=-\omega A_{V} \sin \omega t
$$

The course of the thermal flux has been defined by the relation:

$$
Q(t)=-\frac{4 Q_{\max }}{\tau^{2}} t(t-\tau) \text { for } t \in\langle 0, \tau\rangle
$$

where:

$\mathrm{Q}_{\max }-$ maximal thermal flux for $t \in\langle 0, \tau\rangle$

$\left(\mathrm{Q}_{\max }=5 \mathrm{~kW}\right)$.

The course of temperature and pressure for the assumed excitations $\mathrm{V}(\mathrm{t})$ and $\mathrm{Q}(\mathrm{t})$ is shown on figures 7 and 8 .

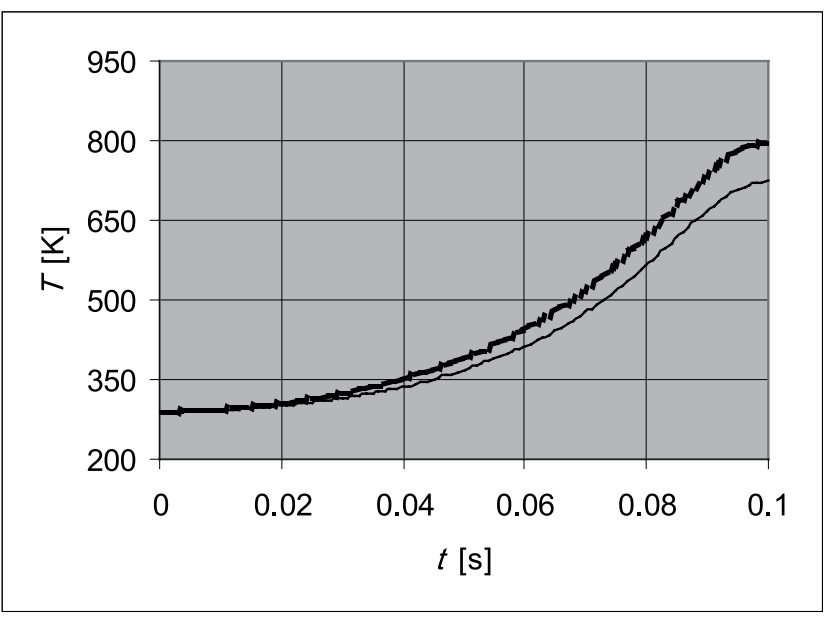

Fig. 7. Course of temperature in the cylinder for the above data: thick linewith heat exchange, according to the relation (12), $Q_{\max }=5 \mathrm{~kW}$; thin line - without heat exchange

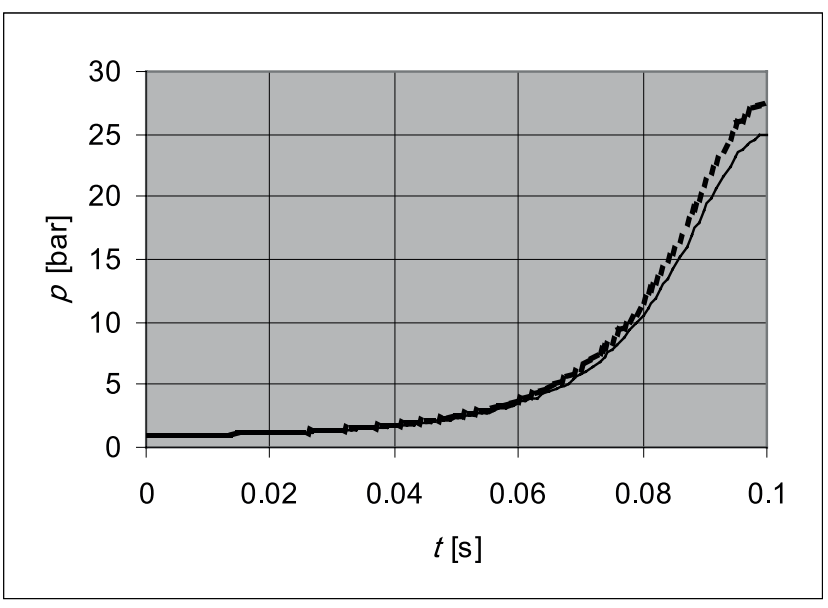

Fig. 8. Course of pressure in the cylinder for the above data: thick line - with heat exchange, according to the relation (12), $Q_{\max }=5 \mathrm{~kW}$; thin line - without heat exchange

It is easy to prove that work without any heat exchange process (presented on fig. 5 and 6) is an adiabatic transformation.

\section{BG MODEL OF THE BASIC ELEMENTS OF HEAT EXCHANGE}

The presented example of modeling encompasses energy transfer processes in the flux of a flowing fluid, heat exchange by convection and conduction as well as dissipation of energy by convection to the environment and by outflow of energy contained in the mass of the flowing medium. A diagram of the system under consideration is shown on figure 9. A model of these processes in the form of a BG, with the elements introduced as part of this approach (fig. 2) being applied is shown on figure 10. 


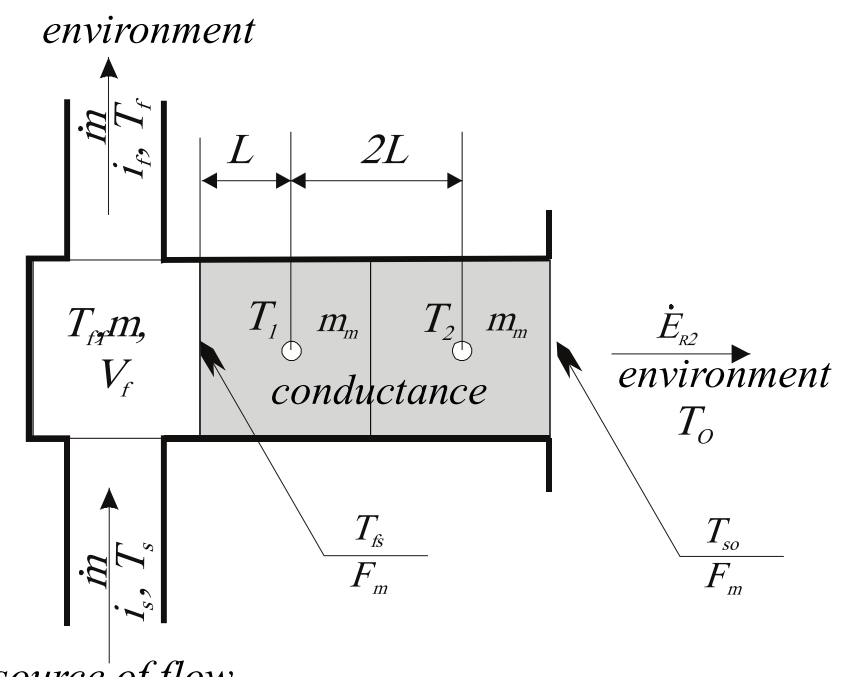

source of flow

Fig. 9. Example of a system of heat exchange by convection and conductance

In accordance with the BG model the state variable vector for the example under consideration is:

$$
\mathbf{T}=\left[\begin{array}{lll}
T_{f} & T_{1} & T_{2}
\end{array}\right]^{T}
$$

The following notations will be introduced for thermal energy flux:

- The energy flux of the source SEFm: $\dot{E}_{S}=\dot{m} \cdot i_{S}$,

- Accumulated energy fluxes $\mathrm{C}_{\mathrm{f}}$ in the liquid heat exchanger: $\dot{E}_{A f}=\dot{m}_{A} ; \mathrm{C}_{1}$ and $\mathrm{C}_{2}$ in the first and second conducting element: $\dot{E}_{A 1}=T_{1} \cdot \dot{S}_{A 1} ; \dot{E}_{A 2}=T_{2} \cdot \dot{S}_{A 2}$,

- $\quad$ Energy flux transferred in the exchanger through the liquid to the first conducting element: $\dot{E}_{f}=\dot{m} \cdot i_{f}=T_{1} \cdot \dot{S}_{11}$,

- $\quad$ Energy flux transferred by the first conducting element to the second conducting element: $\dot{E}_{12}=T \cdot \dot{S}_{12}=T_{2} \cdot \dot{S}_{21}$

- Energy fluxes dissipated by the outflow of the liquid Rol and by convection to the environment $\mathrm{R}_{02}$ : $\dot{E}_{R 1}=\dot{m} \cdot i_{R} ; \dot{E}_{R 2}=T_{2} \cdot \dot{S}_{22}$.

The basic form of state equations obtained directly from the BG model is as follows:

$$
\begin{aligned}
& \dot{E}_{A f}=-\dot{E}_{f}-\dot{E}_{f R o 1}+\dot{E}_{S} \\
& \dot{E}_{A 1}=\dot{E}_{f}-\dot{E}_{12} \\
& \dot{E}_{A 2}=\dot{E}_{12}-\dot{E}_{R o 2}
\end{aligned}
$$

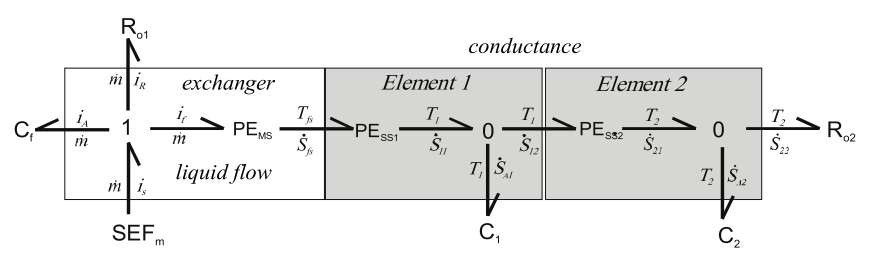

Fig. 10. BG model of the heat exchange processes for the system in figure 6
By substituting the general relation (2) for the speed of thermal energy accumulation and applying the basic convection relations:

$$
\dot{E}_{k o n}=\alpha F\left(T_{H}-T_{L}\right)
$$

and conduction relations:

$$
\dot{E}_{\text {prze }}=\frac{\lambda F_{p}}{L_{p}}\left(T_{i}-T_{i-1}\right)
$$

and by expressing the temperature of the walls $\mathrm{T}_{\mathrm{fs}}$ and $\mathrm{T}_{\mathrm{so}}$ via elements of the vector $T$ and $U$, the form of state equations is obtained (4), which linear form supplemented by algebraic equations is:

$$
\begin{aligned}
& \dot{\mathbf{T}}=\mathbf{A T}+\mathbf{B U} \\
& \mathbf{Y}=\mathbf{C T}+\mathbf{D U}
\end{aligned}
$$

where:

A, B, C and D - matrices whose elements are real numbers,

$\mathrm{Y}$ - the vector of parameters arbitrarily selected for the analysis of parameters (e.g. temperature, heat fluxes, entropy fluxes).

The excitation vector for the case under consideration has the following form in the model (16):

$$
\mathbf{U}=\left[\begin{array}{ll}
T_{s} & T_{o}
\end{array}\right]^{T}
$$

Assuming in this example, that the specific heats, the rate of flow of the fluid and the heat exchange coefficients $\alpha$ and $\lambda$ are constant the state equation is linear and has the form (16). The usage of this method has been also described in $[7,15]$.

\section{EXAMPLE OF NUMERICAL SIMULATION}

For the model shown on figure 9 water has been adopted as the flow medium and aluminum as the conducting material. The model's parameters are contained in table 1. Following transformations and substitution of the parameters stated in table 1 , the following equation matrices were obtained (16):

$$
\boldsymbol{A}=\left[\begin{array}{ccc}
-0.053226 & 0.0120737 & 0.0 \\
0.0123999 & -0.02855 & 0.016151 \\
0.0 & 0.016151 & -0.016226
\end{array}\right]
$$

$$
\boldsymbol{B}=\left[\begin{array}{cc}
0.04115 & 0.0 \\
0.0 & 0.0 \\
0.0 & 7.53 \cdot 10^{-5}
\end{array}\right]
$$


Tab. 1. Model Parameters

\begin{tabular}{|l|l|r|}
\hline $\begin{array}{l}\text { Seq. } \\
\text { No. }\end{array}$ & \multicolumn{1}{|c|}{ Parameter } & Value \\
\hline 1 & Mass of water in the exchanger $m_{f}[\mathrm{~kg}]$ & 0.0729 \\
2 & Mass of the conducting element $m_{m}[\mathrm{~kg}]$ & 0.33125 \\
3 & Length of the conducting element $2 L[\mathrm{~m}]$ & 0.05 \\
4 & Convection exchange area from the liquid side $F_{f}\left[\mathrm{~m}^{2}\right]$ & 0.0025 \\
5 & Convection exchange area from the environment side $F_{o}\left[\mathrm{~m}^{2}\right]$ & 0.0025 \\
6 & Cross section area of the conducting element $F_{p}\left[\mathrm{~m}^{2}\right]$ & 0.0025 \\
7 & Density of water $\rho_{f}\left[\mathrm{~kg} / \mathrm{m}^{3}\right]$ & 972 \\
8 & Density of metal $\rho m\left[\mathrm{~kg} / \mathrm{m}^{3}\right]$ & 2650 \\
9 & Specific heat of water $C_{f}[\mathrm{~J} / \mathrm{kg}]$ & 4200 \\
10 & Specific heat of aluminum $C_{m}[\mathrm{~J} / \mathrm{kg}]$ & 900 \\
11 & Thermal conductivity of aluminum $\lambda_{m}[\mathrm{~W} / \mathrm{mK}]$ & 160.5 \\
12 & Surface conductance from the liquid side $\alpha_{f}\left[\mathrm{~W} / \mathrm{m}^{2} \mathrm{~K}\right]$ & 4000 \\
13 & Surface conductance from the environment side $\alpha_{o}\left[\mathrm{~W} / \mathrm{m}^{2} \mathrm{~K}\right]$ & 15 \\
14 & Mass rate of flow of the liquid $\dot{m}[\mathrm{~kg} / \mathrm{s}]$ & 0.003 \\
\hline
\end{tabular}

Excitation functions (17) were taken to be step functions:

$$
T_{S}=\left\{\begin{array}{l}
293 \text { for } t<0 \\
360 \text { for } t \geq 0
\end{array} ; T_{o}=\left\{\begin{array}{c}
293 \text { for } t<0 \\
293.0001 \text { for } t \geq 0
\end{array}\right.\right.
$$

The following were adopted as the initial conditions for $\mathrm{t}=0$ :

$$
\mathbf{X}_{0}=\left[\begin{array}{lll}
293 & 293 & 293
\end{array}\right]^{T}
$$

Figure 11 shows the course of temperature in individual elements of the system.

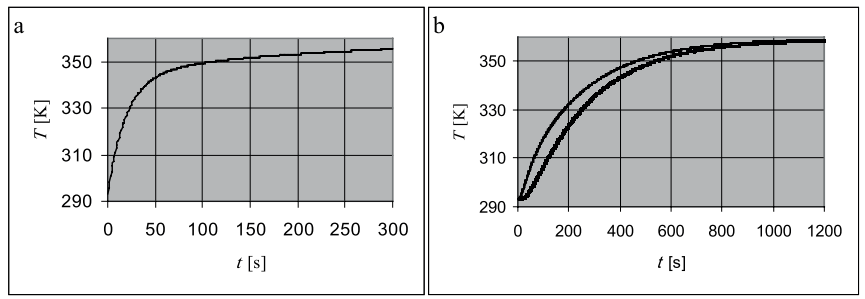

Fig. 11 Course of temperatures: $a$ - in the liquid element;

$b$ - in the metal elements: thin line - 1st, thick line - 2nd element

\section{FINAL CONCLUSIONS}

In this paper, it has been demonstrated that using dual energy storage in the form shown on figure 1 in the modeling of thermal processes by means of bond graphs is not expedient and it is not in line with basic BG theory. The creators of this type of element most probably assumed that there are two forms of accumulating thermal energy. However, according to molecular thermodynamic theory, there is only one form of accumulating thermal energy. In his paper, Anselm [1] formulates the problem thus: "from a molecular point of view, the possibility of changing the system's energy in two ways - by the work carried out on the system and the exchange of heat with the environment becomes self-evident". On the basis of the quoted examples of models of thermodynamic processes and heat exchange, it has been shown that using thermal energy storage in the form of a single node that is applied in basic BG theory for modeling mechanical, hydraulic and electrical processes in BG models of thermal processes is possible and perfectly justified. In the opinion of the Authors, there are no logical arguments that support using so-called "pseudo bond graphs" $[4,10,11,12$, $25,26,27,29]$ for modeling thermal processes, as these do not fulfill the basic condition of the product of the generalized energetic parameters having the same measure as power. It is not possible to agree with some authors [23] adopting entropy as a state variable (in a physical and mathematical sense) that characterizes the accumulation of thermal energy. In accordance with molecular thermodynamic theory it is only temperature that can be such a state variable and this has been adopted in the method of modeling thermal processes presented in this paper. In the proposed method of modeling thermal processes a graph simulating the loss of energy has been adopted as element $\mathrm{R}$.

\section{LITERATURE}

1. Anselm A. I.: Podstawy fizyki statystycznej i termodynamiki. Państwowe Wydawnictwo Naukowe, Warszawa 1980.

2. B. Ould Bouamama K. Medjahera, A.K. Samantarayb, M. Staroswiecki: Supervision of an industrial steamgenerator. Part I: Bond graph modeling. Control Engineering Practice 14 (2006), pp. 71-83.

3. Badoud A.E., et al.: Bond graph modelling and optimization of photovoltaic pumping system. Simulation and experimental results. Simulation Modelling Practice and Theory 36 (2013), pp. 84-103.

4. Borutzki W.: Bond Graph Metodology. Springer Verlag, Erlangen 2010.

5. Borutzki W.: Bond graph modelling and simulation of multidisciplinary systems - An introduction. Simulation Modelling Practice and Theory 17 (2009), pp. 3-21.

6. Burtshtein A. I.: Introduction to Thermodynamics and Kinetic Theory of Matter. John Willey\& Sons, Inc., 1995.

7. Cichy M., Kneba Z., Kropiwnicki J.: Zastosowanie metody grafów wiązań do modelowania układów chłodzenia silników spalinowych. Archiwum Motoryzacji, nr 1, 2005, pp. 21-34.

8. Cichy M.: Modelowanie systemów energetycznych. Wydawnictwo Politechniki Gdańskiej, Gdańsk, 2001.

9. Cichy M.: Nowe podejście do modelowania procesów cieplnych za pomocą grafów wiązań i równań stanu. Prace Naukowe Politechniki Szczecińskiej, Szczecin, 2000.

10. Hubbard M., Brever J. W.: Pseudo Bond Graphs of circulating fluids with Application to Solar Heating Design. Journal of the Franklin Institute, Vol. 311, No. 6, June, 1981. 
11. Karnopp D. C., Margolis D. L., Rosenberg R. C.: System dynamics: a unified approach. Wiley, New York, 1990.

12. Karnopp D.: Pseudo Bond Graphs for Thermal Energy Transport. Journal of Dynamic Systems, Measurement and Control. Vol. 100, Sept. 1978.

13. Kittel C.: Wstęp do fizyki ciała stałego. Wydawnictwo Naukowe PWN, Warszawa, 1999.

14. Kneba Z.: Bond graph modeling of the new generation engine cooling systems. Journal of KONES. Powertrein and Transport, Vol. 13, No. 1, 2006.

15. Kneba Z.: Model chłodnicy płytowej pracującej w układzie chłodzenia samochodowego silnika spalinowego. Archiwum Motoryzacji, nr 1, 2006, pp. 37-45.

16. Kropiwnicki J., Kneba Z., Ziółkowski M.: Test For Assessing the Energy Efficiency of Vehicles with Internal Combustion Engines. International Journal of Automotive Technology, Vol. 14, No. 3 (2013), pp. 479-487.

17. Kurniawan A., et al.: Bond graph modelling of a wave energy conversion system with hydraulic power take-off. Renewable Energy 38 (2012), pp. 234-244.

18. Medjaher K., et al.: Bond graph model of a vertical U-tube steam condenser coupled with a heat exchanger. Simulation Modelling Practice and Theory 17 (2009), pp. 228-239.

19. Mielewczyk A.: A discrete model of the plate heat exchanger. Polish Maritime Research No. 2 (2008), pp. 71-76.

20. Nquien H.: A simulation model of heat exchange in the ship diesel engine cylinder - environment system. Polish Maritime Research No. 1 (2002).

21. Ould Bouamama B.: Bond graph approach as analysis tool in thermofluid model library conception. Journal of the Franklin Institute 340 (2003), pp. 1-23.

22. Pudlik W.: Wymiana i wymienniki ciepła. Politechnika Gdańska, Gdańsk, 1988.

23. Shoureshi R., McLaughlin K. M.: Analytical and Experimental Investigation of Flow-Reversibile Heat Exchangers Using Temperature-Entropy Bond Graphs. Journal of Dynamic Systems, Measurement and Control, Vol. 106, June 1984.

24. Sproull R. L.: Modern Physics. John Willey\& Sons, Inc., New York, Chapman \& Hall, Limited London, 1958.

25. Surjya K. Pal, Derek A. Linkens: Temperature distribution in steel during hot rolling: pseudo-bond graph view. Simulation Modelling Practiceand Theory 10, 2002, pp. 69-85.
26. Thoma J.U., Boumama B.O: Modelling and Simulation in Thermal and Chemical Engineering - a Bond Graph Approach. Springer, 2000.

27. Thoma J.U.: La termodinamica per immagini. Tecniche Nuove, Milano, 1981.

28. Thoma J.U.: Simulation by Bondgraphs. Springer, Berlin, 1990.

29. Tylee J. L. : Pseudo Bond Graph Representation of PWR Pressurizer Dynamics. Journal of Dynamic Systems, Measurement, and Control, Vol. 105, December 1983.

30. Wellstead P. E.: Introduction to System Modelling. Academic Press, London 1979.

\section{CONTACT WITH THE AUTHOR}

$$
\begin{gathered}
\text { M. Cichy } \\
\text { e-mail: mcichy@pg.gda.pl } \\
\text { J. Kropiwnicki } \\
\text { e-mail: jkropiwn@pg.gda.pl } \\
\text { Z. Kneba } \\
\text { e-mail: jkropiwn@pg.gda.pl }
\end{gathered}
$$

Faculty of Mechanical Engineering Gdansk University of Technology Narutowicza 11/12 St. 80-233 Gdańsk POLAND 